\title{
Development of Mathematical Models for Three Phase Medium Transmission Lines
}

\author{
Ramon P. Velasco
}

\begin{abstract}
Engineers, mathematicians, educators, doctors and other professionals are facing the day- to- day challenge of improving individual skills. Engineers are task to develop new technology. Educators are encouraged to develop new teaching methodology. Furthermore, applied mathematicians must contribute new mathematical models that can be used to solve real world problems.
\end{abstract}

Index Terms-Algebraic manipulation, mathematical model, power system, transmission lines.

\section{INTRODUCTION}

Real world problems include security to acquire basic needs such as food, shelter, clothing and education. Electricity plays an important role for security of basic needs. Manufacturing firms use electricity in the production of foods and production of materials for shelter and clothing. Furthermore academic institutions are using electricity for the computers, air conditioners, projectors and new technologies which are essentials to deliver quality education. Hence, electricity is important and a new mathematical model to help us understand electricity is always a welcome development.

"A mathematical model is a description of a system using mathematical concepts and language. The process of developing a mathematical model is termed mathematical modeling. Mathematical models are used in the natural sciences (such as physics, biology, earth science, meteorology), engineering disciplines (e.g. computer science, artificial intelligence) and in the social sciences (such as economics, psychology, sociology and political science). [1]

A concrete example in different fields included the mathematical modeling of material systems (e.g. mass balance of liquid tank, mole balance), thermal systems (heated liquid tank), motion systems (e.g. systems with rotational motion, relations between rotational and linear motion) and mathematical modeling of electrical systems (e.g Kirchhoff's Laws, resulting resistance for series and parallel combination of resistors, mathematical modeling or RC circuit) [2]

Classifications of the mathematical models are: 1) linear or nonlinear 2) deterministic or probabilistic 3) static or dynamic 4) discrete or continuous 5) deductive, inductive or floating. The mathematical model was linear if all variable exhibit linearity, otherwise it is considered nonlinear.

Manuscript received March 6, 2013; revised May 11, 2013.

R. P. Velasco is with Colleges of Arts and Science, Malayan Colleges Laguna, Cabuyao, Laguna Philippines (e-mail:bseerv@yahoo.com/rpvelasco@mcl.edu.ph). represented with non-linear equation it said to be non-linear. [1]

The objectives of creating a mathematical model are the following: 1) to explain a phenomenon, 2) to make predictions about behavior, 3) decision making. Unfortunately there are some limitations of the model specifically in the physical system as a completely precise model of a physical system is not possible. There are always phenomena which will not be able to model that contribute to model errors or uncertainty. The important thing is the model describes the most important properties of the system. [1], [3]

In addition, the quality of a scientific field depends on how well the mathematical models developed on the theoretical side agree with results of repeatable experiments. Lack of agreement between theoretical mathematical models and experimental measurements often leads to important advances as better theories are developed. A classic example is that when the planetary model developed by Ptolemy was replaced by a superior and simple planetary model of Kepler. [1]

Developing a mathematical model follow the following procedure: 1) define system boundaries, 2) make simplifying assumptions, 3) use the balance law for the physical system, and define eventual additional conditions, 4) drawn an overall block diagram showing inputs, outputs and the parameters, 5) present the model in proper form. [1]

Today physicists, engineers, statisticians, operations research analysts and economists use mathematical model to improve the life of many individual. Engineers used their knowledge on mathematical model in their respective field to explain phenomenon, make predictions, in decision making, to solve real word problems and many other things. Mathematical model if properly understood is a great tool to solve a lot of work related problem. [1]

In the Philippines, the mathematical model applicable in the engineering is taught at the higher level institution. The mathematical model is clearly explained in the class room. Students appreciate the model much better when applied to a real world scenario. The advantage of engineering is that the model can be verified by the students using actual laboratory activity.

The main objective of the study is to develop new mathematical models for a three phase medium transmission line with unbalance shunt admittance. The specific objectives are the following:

1) to develop a mathematical model that can express source voltage in terms of receiving voltage and receiving current

2) to develop a mathematical model that can express source current in terms of receiving voltage and receiving current 
For the scope and limitation, the study covers the derivation of a new mathematical model that will show the relationship of voltage and current at the sending and receiving end of a three phase transmission line.

For the mathematician, the research will show the importance of mathematics as foundation of engineering subjects.

In conclusion, the newly developed mathematical model will be beneficial for the engineering students, engineers and engineering educators in solving real world problems. It is time to replace the three phase medium transmission line with balance shunt admittance model for a much more beneficial model that can be used to analyze medium transmission system satisfying both balance and unbalance shunt admittance. Hence the study is conducted.

\section{POWER SYSTEM}

Power system is the branch of electrical engineering specialized in the design, operation, maintenance and analysis of the generating stations, transmission lines and distribution lines. Transmission lines interconnect the generating stations and the distribution lines. Four parameters of electrical transmission lines are resistance, inductance, capacitance and conductance. Overhead transmission lines classification are 1) short transmission lines which is less than $80 \mathrm{~km}$ long, 2) medium transmission lines which are between $80 \mathrm{~km}$ to $240 \mathrm{~km}$, 3) long transmission lines which are longer than $240 \mathrm{~km}$. [3]-[6].

A short transmission lines approximation usually neglected shunt capacitance. The mathematical model of short transmission lines is derived using Kirchhoff's law and analysis of the current in series circuit.

The mathematical models for short transmission lines per phase are shown in equation 1 and 2. Fig. 1 illustrated the circuit diagram of a short transmission lines. [3]-[6].

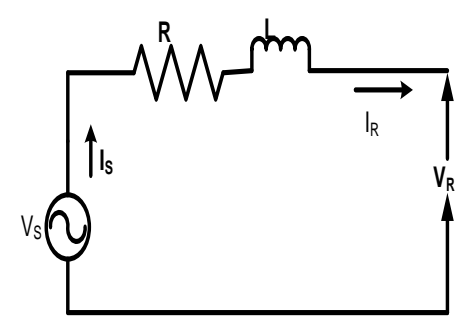

Fig. 1. Circuit diagram of short transmission line per phase.

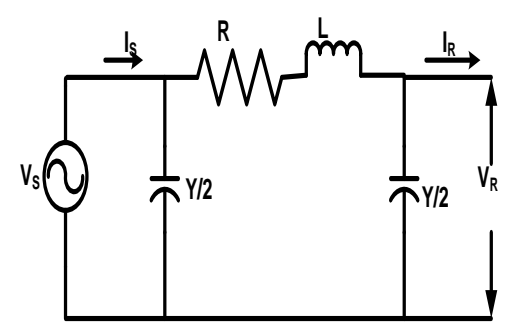

Fig. 2. Circuit diagram of medium transmission line pre phase.

$$
\begin{gathered}
I_{s}=I_{r} \\
V_{s}=V_{r}+I_{r} Z
\end{gathered}
$$

Medium transmission lines approximation usually considered pure capacitance as shunt admittance. The model was derived using Kirchhoff's law, Ohms law and algebraic manipulation. [8], [9]

The mathematical models for a medium transmission lines per phase are shown in (3) and 4. Fig. 2 illustrated the circuit diagram of a short transmission lines. [3]-[7]

$$
\begin{gathered}
V_{s}=A V_{r}+B I_{r} \\
I_{s}=C V_{r}+D I_{r} \\
A=D=\frac{Z Y}{2}+1 \\
B=Z \\
C=Y\left(1+\frac{Z Y}{4}\right)
\end{gathered}
$$

where:

$V_{s}$ is the sending voltage $(\mathrm{V})$

$V_{r}$ is the receiving voltage $(\mathrm{V})$

$I r$ is the receiving current (A)

$I_{s}$ is the sending current (A)

$R$ is the resistance of transmission line $(\Omega)$

$L$ is the inductance o transmission line $(\mathrm{H})$

$Y$ is the shunt admittance (mho)

$\mathrm{A}, \mathrm{B}, \mathrm{C}$ and $\mathrm{D}$ are transmission lines constant

\section{DiscusSIONS AND RESUltS}

\section{A. Derivation of the Mathematical Model of Expressing Source Voltage in Terms of Receiving Voltage and Receiving Current}

Step 1. The researcher used an unbalance $\pi$ circuit. Fig. 3 illustrated the circuit diagram of short transmission line per phase.

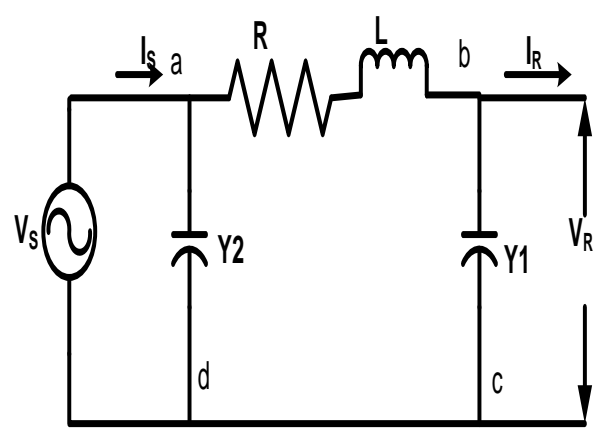

Fig. 3. Circuit diagram of short transmission line per phase.

Step 2. In medium transmission a pure value of capacitance is considered for the shunt admittance. The source resistance is neglected to simplify the calculations. The following representations are used in relation to electrical parameters demonstrated in the preceding circuit. 
$V_{s}$ is the sending voltage $(\mathrm{V})$

$V_{r}$ is the receiving voltage $(\mathrm{V})$

$I_{r}$ is the receiving current (A)

$I_{s}$ is the sending current (A)

$R$ is the resistance of transmission line $(\Omega)$

$L$ is the inductance o transmission line $(\mathrm{H})$

$Y_{1}$ is the shunt admittance in the right side of the model (mho)

$Y_{2}$ is the shunt admittance in the right side of the model (mho)

Step 3. Use Kirchhoff's law, and Ohm's law and algebraic manipulation such as substitution, simplifying equation, distribution, grouping of similar terms and factoring will also be used to present the model in proper form.

Apply Kirchhoff's Voltage Law at loop abcd

$$
V_{s}=V_{r}+V_{z}
$$

Using Ohm's Law

$$
V_{z}=I_{z} Z
$$

Substitute equation 9 to 8

$$
V_{s}=V_{r}+I_{z} Z
$$

Using Kirchoff's current Law at node B

$$
I_{z}=I_{Y 1}+I_{r}
$$

But using Ohm's Law

$$
I_{Y 1}=V_{r} Y_{1}
$$

Substitute equation 12 to 11

$$
I_{z}=V_{r} Y_{1}+I_{r}
$$

Substitute equation 13 to 10

$$
V_{s}=V_{r}+\left(V_{r} Y_{1}+I_{r}\right)(Z)
$$

Application of Distributive Property to 14

$$
V_{s}=V_{r}+Z V_{r} Y_{1}+Z I_{r}
$$

Group Similar Terms

$$
V_{s}=\left(V_{r}+Z V_{r} Y_{1}+Z I_{r}\right)
$$

Factor GCF

$$
V_{s}=V_{r}\left(1+Z Y_{1}\right)+Z I_{r}
$$

Apply substitution

Let $E=\left(1+Z Y_{1}\right)$
Let $F=Z$

Substitute A and B to equation 8

$$
V_{s}=E V_{r}+F I_{r}
$$

Step 4. Hence the first mathematical model is

$$
V_{s}=E V_{r}+F I_{r}
$$

Step 5. Draw an overall black diagram.

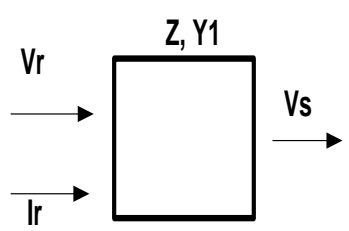

B. Derivation of the Mathematical Model of Expressing Source Current in Terms of Receiving Voltage and Receiving Current

STEP 1, 2 and 3 are repeated.

Applying Kirchoff's Current Law

$$
I_{s}=I_{Y 2}+I_{r}+I_{Y 1}
$$

Using Ohms’s Law

$$
\begin{gathered}
I_{Y 1}=V r Y_{1} \\
I_{Y 2}=V_{s} Y_{2}
\end{gathered}
$$

Substitute (19) and (20) to (18)

$$
I_{s}=V_{s} Y_{2}+I_{r}+V_{r} Y_{1}
$$

Substitute $V_{s}=V_{r}\left(1+Z Y_{1}\right)+Z I_{r}$ to (21)

$$
I_{s}=\left(V_{r}\left(1+Z Y_{1}\right)+Z I_{r}\right) Y_{2}+I_{r}+V_{r} Y_{1}
$$

Apply Distributive Property

$$
I_{s}=V_{r} Y_{2}\left(1+Z Y_{1}\right)+Z I_{r} Y_{2}+I_{r}+V_{r} Y_{1}
$$

Apply GCF and recall that $\mathrm{E}=\left(1+Z Y_{1}\right)$

$$
\begin{aligned}
& I_{s}=V_{r} Y_{2}\left(1+Z Y_{1}\right)+\operatorname{Vr} Y_{1}+Z I_{r} Y_{2}+I_{r} \\
& I_{s}=\operatorname{Vr}\left[Y_{2}\left(1+Z Y_{1}\right)+Y_{1}\right]+\operatorname{Ir}\left(Z Y_{2}+1\right) \\
& I_{s}=\operatorname{Vr}\left[E Y_{2}+Y_{1}\right]+\left(Z Y_{2}+1\right) I_{r}
\end{aligned}
$$

Apply Substitution and let $\mathrm{G}=E Y_{2}+Y_{1}$ and $\mathrm{H}=$ $Z Y_{2}+1$

$$
I_{s}=G V_{r}+H I_{r}
$$


Step 4. Hence the second mathematical model is

$$
I_{s}=G V_{r}+H I_{r}
$$

Step 5. Draw an overall black diagram

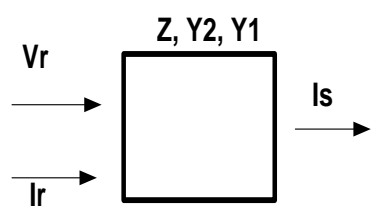

\section{CONCLUSION}

The mathematical models for a three phase medium transmission lines $V_{s}=E V_{r}+F I_{r}$ and $I_{s}=G V_{r}+H I_{r}$ are developed using Kirchhoff's law, Ohm's Law and algebraic manipulation. The derivation of the two models demonstrated the importance of Physics and Mathematics in mathematical modeling.

Furthermore, the two mathematical models are effective in predicting the value of the source voltage and source current given the receiving voltage and receiving current of a three phase medium transmission lines.

\section{ACKNOWLEDGMENT}

The registration of the paper is supported by Malayan Colleges Laguna. I would like to thank Dr. Florencio Reyes my thesis adviser for sharing his expertise in this study. I would like to thank Dr. Ricardo Bobadilla, Dr. Luisito Hagos and Dr. Vicente Castillo for being the best critique. I would to thank all my superiors and colleagues in Malayan Colleges
Laguna for all the support. This work is dedicated to my wife Leizle, my daughter Lira Samantha and son Edward James for all the inspiration and motivation.

\section{REFERENCES}

[1] Mathematical model. (July 9, 2012). [Online]. Available: http://en.wikipedia.org/wiki/Mathematical_model.

[2] Mathematical modeling, Techteach Mathematical Modeling, chapter 3, July 15, 2012.

[3] M. E. Hawaray, Introduction to electrical power systems, $1^{\text {st }}$ edition, New Jersey, USA: John Wiley and Sons, 2008, chapter 5, pp. 176-182.

[4] B. M. Weedy and B. Cory, Electric power system, $4^{\text {th }}$ Ed., England: John Wiley and Sons, chapter 3, 1998, pp. 122-128.

[5] D. P. Kothari and I. J. Nagrath, Power system engineering, $2^{\text {nd }}$ Ed., New Delhi: Tata Mc Graw-Hill Publishing Company, chapter 5, 2008, pp. 176-186.

[6] S. A. Nasar, Schaum's outline for electric power system, $1^{\text {st }}$ Ed., USA: McGrawHill, chapter 3, 1990, pp. 26-29.

[7] W. Stevenson, Elements of power system analysis, $4^{\text {th }}$ Ed., McGraw-Hill Book Company, chapter 5, 1984, pp. 88-93.

[8] M. Schaultz, Grobs basic electronics, $1^{\text {st }}$ edition, New York, USA: Mc Graw Hill Higher Education, chapter 9, 2007, pp. 270-280.

[9] R. L. Boylestad, Introductory to circuit analysis, $11^{\text {th }}$ Ed., New Jersy, USA: Pearson Education, chapter. 15, 2007, pp. 637-697.

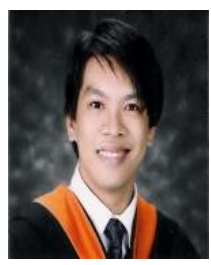

Ramon P. Velasco was born in the Philippines on April 9, 1984. He finished his bachelor of science in electrical engineering at Colegio de San Juan de Letran- Calamba in 2000 and master of science major in mathematics at University of Cabuyao (Pamantasan ng Cabuyao-2013). The author has also worked as manufacturing engineer in Samsung Electronics in 2006 and cirtek electronics in 2007. Today, he is presently connected with Malayan Colleges Laguna (MCL) a subsidiary of Mapua Institute of Technology (MIT) one of the leading engineering institution in the Philippines. The author is teaching Mathematics, Physics and professional courses in Electrical engineering subjects. The author research interest is mathematical modeling related to electricity. 